\title{
Efecto del piso altitudinal sobre la calidad de la mora (Rubus glaucus benth) en la región interandina del Ecuador
}

\author{
Effect of the altitudinal floor on the quality of mora (Rubus glaucus benth) in the \\ interand region of Ecuador \\ José Antonio Sánchez-Morales ${ }^{1}$; Marlon Xavier Villares-Jibaja1 ; Zulay Niño-Ruiz ${ }^{*}$; Maria B. \\ Ruilova ${ }^{1}$
}

\begin{abstract}
RESUMEN
La mora de castilla (Rubus glaucus Benth) contribuye al sustento económico de cerca de 12000 familias de medianos y pequeños productores en el Ecuador pero la baja productividad de las plantaciones y la falta de calidad de la fruta, limitan las posibilidades de mejorar los ingresos y de comercializar de manera justa el producto. El escaso conocimiento de las consecuencias de las zonas agroclimáticas sobre el cultivo contribuye a este panorama. En esta investigación se planteó estudiar el efecto sobre el cultivo de Mora de Castilla de tres pisos altitudinales de la provincia Bolívar, Ecuador. Se procedió a la selección de plantas con mayor producción y menores problemas fitosanitarios, en época seca y en conjunto con los productores. Se seleccionaron cultivos ubicados tres alturas diferenciadas y se realizó una caracterización fenológica y fitopatológica in situ de dichas plantas, estudiando 3 tratamientos productores por zona, con 5 repeticiones. Para determinar diferencias de productividad entre las plantas de las tres zonas se realizó un análisis de varianza y prueba de Tukey al $5 \%$. Se observa variaciones significativas en el número de tallos terciarios y en el número de hojas, entre las plantas ubicadas en el piso altitudinal alto y bajo, lo que permite inferir un aumento de la productividad de los huertos de este último piso altitudinal. Igualmente los días transcurridos de la floración a la cosecha son menores en el piso altitudinal bajo, lo que permitiría mayores ciclos de producción por año, con su consecuente aporte económico.

Palabras clave: Piso altitudinal, variables fenológicas, problemas fitosanitarios, productividad, Provincia Bolívar.
\end{abstract}

\begin{abstract}
Cultivation of the Andean Raspberry (Rubus glaucus Benth) contributes to the economic livelihood of nearly 12000 medium and small-scale producer families in Ecuador, however the low productivity of this crop and lack of quality of its fruit limit the possibilities of increasing income and fair commercialization of the product. Little knowledge about the effect of agro-climatic zones on the cultivation of this crop contributes to this situation. This research's aim was to study the effect of three altitudinal levels, in Bolivar Province, Ecuador, on the harvesting of the Andean Raspberry. Plants with higher production and less phytosanitary problems, during the dry season, where selected in cooperation with local producers. Crops from three different altitudes were selected and subjected to in situ phenological and phytopathological characterization, studying 3 producers per zone, with 5 repetitions. An analysis of variance and Tukey test at $5 \%$ were conducted in order to determine productivity differences between plants from the three zones. Significant variations were observed in the number of tertiary stems and number of leaves between plants located in the high and low altitudinal levels. This makes it possible to infer a productivity increase in the orchards located in the lowest altitudinal level. Days transcurred between blooming and harvest are also less in the low altitudinal level, which allows for more production cycles per year and consequently a higher revenue for small-scale producers of this berry.
\end{abstract}

Key Words: agro-climatic zone, phenological variables, phytosanitary problems, productivity, Bolivar Province

\section{Introducción}

La mora de castilla (Rubus glaucus Benth), es un frutal andino que pertenece a la familia Rosacea, genero Rubus que agrupa aproximadamente 700 especies a nivel mundial (Yih \& Ming 2009). Esta especie está ampliamente distribuida en América Central, México y en América del sur (Franco \& Giraldo, 2000). Según Serna et al. (2009), es considerada una especie frutícola con

1 Universidad Estatal de Bolívar UEB. Facultad de Ciencias Agropecuarias. Recursos Naturales y del Ambiente. Campus Académico "Alpachaca" Av. Ernesto Che Guevara s/n y Av. Gabriel Secaira, C.P. 020150, Guaranda, Ecuador.

* Autor por correspondencia: znino09@gmail.com; znino@ueb.edu.ec

Fecha de Recepción: 07 marzo, 2018.

Fecha de Aceptación: 02 mayo, 2018.

DOI: http://dx.doi.org/10.4067/S0718-34292018005000702. Publicado en línea: 06-septiembre-2018. 
un alto potencial de demanda, que actualmente es comercializada en el Ecuador en el mercado nacional para consumo en fresco e industrializado, y en el internacional en pulpa y extracto, fundamentalmente hacia los Estados Unidos. Estudios realizados en Colombia en el año 2009 indican un potencial de crecimiento anual del orden de 12\% (Barrero, 2009).

En el Ecuador la mora tiene gran importancia socioeconómica, debido a su capacidad productiva en pequeñas áreas, se cultiva en las provincias de Tungurahua, Cotopaxi, Bolívar, Chimborazo, Pichincha, Imbabura, Carchi (MAGAP, 2013 y Martínez et al. 2007). Soria, Padilla \& Larrea (2014), reportan que existe una superficie aproximada de 5247 ha, en 14546 Unidades productivas agricolas (UPAs); distribuidas en las zonas agroclimáticas desde 2000 hasta $2800 \mathrm{msnm}$, permitiendo el sustento económico y permanente de más de 12000 familias de medianos y pequeños productores, además que este cultivo genera ingresos económicos permanentes, a diferencia de otros rubros como maíz o fréjol cuya producción y réditos económicos son anuales (MAGAP, 2013).

Según Ayala, Valenzuela y Bohorquez (2013a) la mora de castilla es un fruto de vida muy corta con compuestos bioactivos. Estos frutos enfrentan continuos cambios fisicoquímicos que afectan su demanda, además de ser fácilmente afectados por Botrytis Cinerea (Ayala, Valenzuela y Bohorquez, 2013a y 2013b). Estos autores reconocen la importancia de evaluar las características del fruto en diferentes zonas agroclimáticas, al igual que Cancino et al (2011) quienes indican que en Colombia, este cultivo no ha alcanzado el grado de desarrollo deseado, debido a la falta de un sustento tecnológico adecuado y a la falta de esfuerzos de mejoramiento.

Valdés et al. (2012) realizó estudios de caracterización agronómica y molecular de especies vegetales fundamentales en programas de mejoramiento genético, enfocados al desarrollo de nuevos cultivares, lo que permite, según Franco et al. (2000) medir la variabilidad genética e identificar genes especiales, denominados 'stocks genéticos' que son utilizados para investigaciones, como es el caso de la resistencia a factores bióticos y abióticos.

Por su parte Moreno et al. (2011), realizaron caracterización morfológica in situ de mora (Rubus spp) de 65 individuos que correspondían a las especies: Rubus urticifolius, $R$. glaucus, $R$. bogotensis, $R$. macrocarpus, $R$. niveus, $R$. robustus y un material que no fue posible su clasificación taxonómica, encontrando en el análisis de clasificación tres grupos donde el mayor aporte a la variabilidad fue: color de corola, pubescencia de tallo, forma de infrutescencia, forma de tallo y forma de estípulas.

En Ecuador, Garrido, Vásquez y Morillo, (2010) analizaron la diversidad genética de 108 accesiones de mora cultivada y especies emparentadas, provenientes de las provincias de Cotopaxi, Tungurahua, Bolívar, y Loja en el Ecuador, incluyendo muestras de accesiones colombianas conservadas por el Instituto Nacional de Investigaciones Agropecuarias (INIAP) mediante el uso de marcadores moleculares, determinándose que en Ecuador se cultiva únicamente la especie estudiada. Sin embargo, aún no se realizan estudios que permitan determinar el efecto de la zona agroclimática tal como el realizado en Colombia por Alzate, Mayor y Montoya (2010) en el Departamento de Caldas donde no observaron diferencias considerables entre las condiciones climáticas generales de las dos zonas estudiadas.

Porotrolado,Lemus y Ramírez(2002) analizaron la fenología reproductiva en tres tipos de vegetación de la planicie costera de la península de Paraguaná, Venezuela, encontrando que las variaciones estacionales en la fenología reproductiva presentaron un máximo que coincidió con los valores mínimos de precipitación, tres meses después del máximo de precipitación, lo que sugiere que la cantidad de precipitación asociada a los pisos altitudinales puede influir en la productividad del cultivo analizado.

La provincia Bolívar, Ecuador está situada en el centro - oeste del país en las coordenadas UTN 9872857 hasta 9756351 metros Norte y 678594 hasta 742762 metros Este. En esta provincia existen zonas de vida de acuerdo a la clasificación de L. Holdrige (1.967), del tipoforrmación bosque montano bajo, identificados en sectores de transición al subtrópico. Se diferencian tres pisos altitudinales: alto, medio y bajo; donde el piso alto se caracteriza por tempraturas promedio de $12.3{ }^{\circ} \mathrm{C}$ con precipitaciónes de 873 $\mathrm{mm} / \mathrm{año}$; el piso medio por temperaturas promedio de $13.7^{\circ} \mathrm{C}$ y precipitaciónes de $869 \mathrm{~mm} / \mathrm{año} \mathrm{y} \mathrm{el}$ piso bajo con temperaturas promedio de $16{ }^{\circ} \mathrm{C}$ y precipitaciónes de $1151 \mathrm{~mm} /$ año.

En este sentido resulta esencial continuar con investigaciones para determinar la mejor zona para el cultivo de mora, lo que ayuda a la identificación y selección de plantas con mayor producción y menores problemas fitosanitarios, lo que según Phillips et al. (2012), permitirá la formación de jardines clónales como fuente de material vegetativo. En este trabajo 
se evaluó el efecto del piso altitudinal en el desarrollo fenológico del cultivo de R. glaucus, con la finalidad de identificar en cual de estas zonas existen plantas que posean características deseables tanto de resistencia a factores bióticos y abióticos como de calidad, para consumidores de fruta fresca y para la agroindustria.

\section{Materiales y métodos}

\section{Definición de las zonas agroclimáticas para seleccionar las plantas a estudiar}

Se visitó, en el mes de junio 2016, inicio de la época seca, zonas productoras de mora de la provincia Bolívar, como son Nina Rumi, San Francisco, Cerritos, Matapalo, Jashi, Arrayampamba en el cantón Chillanes, Capilluco, Illubi, Guantug Cruz en el cantón Guaranda y La Magdalena, Gualasay, Tiumbucho en el cantón Chimbo, registrando datos de altitud sobre el nivel del mar, posición y características de los cultivos de mora de cada lugar, con la finalidad de seleccionar en tres pisos altitudinales: alto (A) de 2500 a 3000 m.s.n.m, medio (M) con altitud superior a 2100 e inferior a 2500 m.s.n.m y bajo (B) con altitud inferior a 2100 m.s.n.m, fincas que presenten el cultivo de la especie estudiada y el mismo manejo agronómico para evitar las desviaciones expresadas por Alzate, Mayor y Montoya (2010). Para la determinación taxonómica se procedió según lo indicado por Cancino-Escalante et al. (2011). La ubicación geográfica de los sectores seleccionados se muestra en la figura 1. El piso altitudinal alto correspondió a Guantug Cruz; el piso altitudinal medio se encontró en San Francisco, Nina Rumi y Matapalo $\mathrm{y}$ el piso altitudinal bajo en Capilluco.

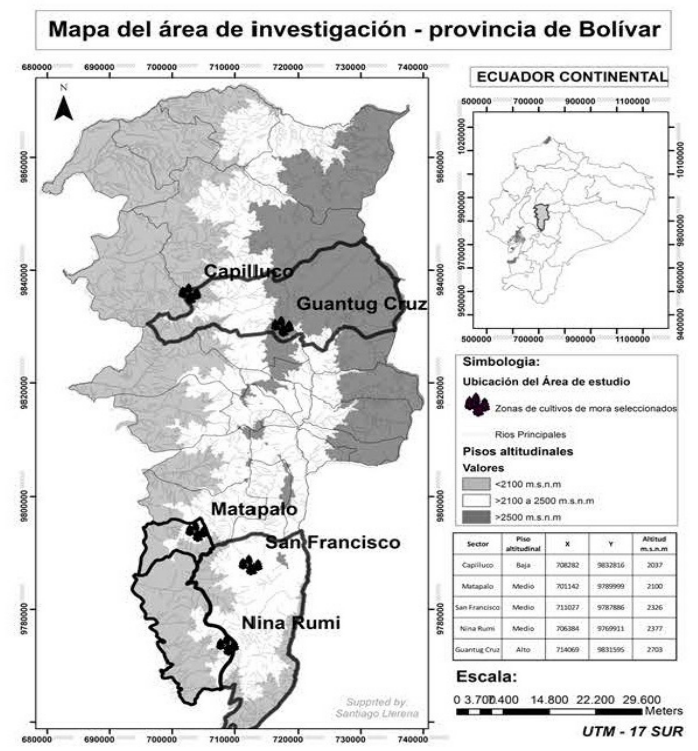

Figura 1. Ubicación de los huertos de plantas de mora de la variedad castilla donde se realizó la investigación.

Una vez seleccionadas las zonas, se procedió a identificar 5 fruticulturas que disponían huertos de mora de la variedad castilla (Rubus glaucus), se identificaron 3 plantas por huerto con características superiores; considerando como superiores aquellas plantas que según el productor tenían mayor vigor, menor incidencia de plagas y enfermedades y el fruto de mayor volumen a lo largo de la vida de la planta. Así se seleccionaron 15 plantas en cada zona agroclimática. La investigación se realizó en los meses de junio a octubre, que representa época seca.

\section{Caracterización fenotípica de las plantas seleccionadas}

Para caracterizar la variabilidad fenotípica de los huertos de mora, se evaluaron variables fenológicas (diámetro de copa, vigor de planta, longitud de la hoja, diámetro de hoja, \# de frutos por centros de 
producción, peso del fruto, rendimiento, longitud del fruto, diámetro del fruto, acidez titulable y solidos solubles) y variables fitopatológicas (incidencia de
Botrytis, Oídio, Peronospora y otros). Todas las variables se determinaron como se indica en la Tabla 1.

Tabla 1. Variables experimentales fenológicas, fitopatológicas y forma de determinarlas

\begin{tabular}{|c|c|c|}
\hline Parámetro & Unidades & Forma de medición* \\
\hline Diámetro de la copa & $\mathrm{cm}$ & Con una cinta métrica, a los $2 / 3$ de altura de plantas. \\
\hline Vigor de la planta & adm & $\begin{array}{l}\text { Se utilizó una escala hedónica siendo } 1 \text { lo más débil y } 5 \\
\text { las plantas de mayor vigor. }\end{array}$ \\
\hline $\begin{array}{l}\text { Número total de tallos } \\
\text { principales }\end{array}$ & adm & $\begin{array}{l}\text { Se consideraron todos los tallos que emergen a partir del } \\
\text { cuello radicular. }\end{array}$ \\
\hline $\begin{array}{l}\text { Número total de tallos } \\
\text { secundarios }\end{array}$ & $\mathrm{adm}$ & $\begin{array}{l}\text { Se consideraron todos los tallos que emergen de un tallo } \\
\text { principal. }\end{array}$ \\
\hline $\begin{array}{l}\text { Número total de tallos } \\
\text { terciarios }\end{array}$ & adm & $\begin{array}{l}\text { Se consideraron todos los tallos que surgen de un tallo } \\
\text { secundario. }\end{array}$ \\
\hline $\begin{array}{l}\text { Número total de } \\
\text { racimos }\end{array}$ & adm & $\begin{array}{l}\text { Se consideraron todos los racimos que emergen de los } \\
\text { tallos secundarios y terciarios. }\end{array}$ \\
\hline Longitud de la hoja & $\mathrm{mm}$ & $\begin{array}{l}\text { Con un flexómetro se mide la longitud y diámetro en } \\
\text { (mm) de } 5 \text { hojas tomadas de la porción media de dos } \\
\text { ramificaciones por planta. }\end{array}$ \\
\hline Diámetro de la hoja & $\mathrm{mm}$ & $\begin{array}{l}\text { Con un flexómetro se mide la longitud y diámetro en } \\
(\mathrm{mm}) \text { de } 5 \text { hojas tomadas de la porción media de dos } \\
\text { ramificaciones por planta. }\end{array}$ \\
\hline \# de frutos por racimo & adm & $\begin{array}{l}\text { Se contaron el número de frutos presentes en un eje de } \\
\text { producción seleccionado al azar. }\end{array}$ \\
\hline Peso del fruto & $\mathrm{g}$ & $\begin{array}{l}\text { Se seleccionaron } 5 \text { frutos a la madurez fisiológica en } \\
\text { dos ramificaciones por planta y se pesaron en Balanza } \\
\text { digital marca Diamont Modelo } 500 \text { (rango: } 0.1 \mathrm{~g}-500 \\
\text { g). }\end{array}$ \\
\hline Rendimiento & $\mathrm{kg} /$ planta & $\begin{array}{l}\text { Se cuantificó el rendimiento y luego el número de } \\
\text { plantas/ha, generando ton/ha. }\end{array}$ \\
\hline Longitud del fruto & $\mathrm{mm}$ & Se midió con un calibrador digital marca Stanly. \\
\hline Diámetro del fruto & $\mathrm{mm}$ & Se midió con un calibrador digital marca Stanly. \\
\hline Forma del fruto & adm & $\begin{array}{l}\text { Se dividió la longitud para el diámetro del fruto, si el } \\
\text { resultado es mayor a } 1 \text { la forma es cónica, si el resultado } \\
\text { es igual o inferior a } 1 \text { la forma es redondeada. }\end{array}$ \\
\hline Acidez titulable & $\%$ & Análisis de titulación con hidróxido de sodio $0.1 \mathrm{~N}$. \\
\hline Solidos solubles & $\%$ & $\begin{array}{l}\text { Porcentaje de grados Brix utilizando un refractómetro } \\
\text { marca. Ref } 113 \text { brix/atc } 0-32 \% \text {. }\end{array}$ \\
\hline Días totales de ciclo & días & $\begin{array}{l}\text { Se contabilizó el número de días transcurridos de yema } \\
\text { hinchada en donde el botón se encuentra cerrado y } \\
\text { presenta un color verde café, a fruto fisiológicamente } \\
\text { maduro que presenta un color negro rojizo. }\end{array}$ \\
\hline $\begin{array}{l}\text { Incidencia de plagas y } \\
\text { enfermedades }\end{array}$ & $\%$ & $\begin{array}{l}\text { Se seleccionaron cinco ramas por planta y en cada } \\
\text { una de ellas se contabilizó el número de flores y frutos } \\
\text { que presentaron la enfermedad en las inflorescencias } \\
\text { formadas (Ayala et al. (2013b). El porcentaje de } \\
\text { Incidencia se evaluó como cantidad de frutos enfermos/ } \\
\text { cantidad del total de frutos x } 100 \text {. Con una severidad } \\
\text { baja }<10 \% \text {, media } 10 \text { a } 25 \% \text { y alta }>30 \% \text {. }\end{array}$ \\
\hline
\end{tabular}

${ }^{*}$ Los descriptores y variables evaluadas se tomaron de Cancino et al (2011) y Ayala, Valenzuela y Bohorquez (2013b ) 


\section{Análisis estadístico}

Para determinar diferencias entre zonas agroclimáticas se realizó un análisis de varianza para todas las variables evaluadas y una prueba de Tukey al $5 \%$ mediante el software Statistix 9.0.

\section{Resultados y discusión}

\section{Caracterización fenotípica}

La tabla 2 muestra los resultados de las variables fenológicas estudiadas en los tres pisos altitudinales.

Según los resultados de la prueba de Tuckey al 5\%, las variables diámetro de copa, vigor de planta, número de tallos principales, numero de tallos secundarios, número de racimos, longitud de la hoja, diámetro de hoja, son estadísticamente iguales en las tres zonas agroclimáticas estudiadas, mientras que el número de tallos terciarios y número de hojas es estadísticamente mayor en el piso altitudinal bajo, que en el alto. Grijalba, Calderón y Pérez (2010) obtuvieron un promedio de 5 tallos basales por planta, lo cual es significativamente inferior a los resultados obtenidos en este estudio. Bernal y Díaz (2006) asociaron que a mayor número de tallos mayor producción del cultivar, lo que permite inferir que la productividad es mayor en el piso altitudinal bajo por tener un mayor número de tallos terciarios.

Tabla 2. Valores promedio de las variables evaluadas en la caracterización fenológica de mora (R. glaucus) en los tres pisos altitudinales estudiados.

\begin{tabular}{|c|c|c|c|c|}
\hline \multirow{2}{*}{ Variable } & \multicolumn{3}{|c|}{ Piso altitudinal } & \multirow{2}{*}{ P-value } \\
\hline & Alto & Medio & Bajo & \\
\hline Diámetro de copa & $\begin{array}{c}2.91^{\mathrm{a}} \\
(0.52)\end{array}$ & $\begin{array}{r}3.41^{\mathrm{a}} \\
(0.80)\end{array}$ & $\begin{array}{c}3.01^{\mathrm{a}} \\
(0.30)\end{array}$ & 0.0902 \\
\hline Vigor de planta & $\begin{array}{r}4.10^{\mathrm{a}} \\
(0.38)\end{array}$ & $\begin{array}{r}4.17^{\mathrm{a}} \\
(0.56)\end{array}$ & $\begin{array}{r}4.40^{\mathrm{a}} \\
(0.50)\end{array}$ & 0.2692 \\
\hline \# de tallos Principales & $\begin{array}{r}19.87^{\mathrm{a}} \\
(3.81)\end{array}$ & $\begin{array}{r}19.73^{\mathrm{a}} \\
(5.63)\end{array}$ & $\begin{array}{r}22.47^{\mathrm{a}} \\
(6.45)\end{array}$ & 0.3172 \\
\hline \# tallos Secundarios & $\begin{array}{l}6.00 \mathrm{a}^{\mathrm{a}} \\
(2.39)\end{array}$ & $\begin{array}{r}6.93^{\mathrm{a}} \\
(2.08)\end{array}$ & $\begin{array}{c}6.00^{\mathrm{a}} \\
(1.19)\end{array}$ & 0.3057 \\
\hline \# de tallos Terciarios & $\begin{array}{c}3.33^{\mathrm{b}} \\
(1.04)\end{array}$ & $\begin{array}{c}4.47^{\mathrm{ab}} \\
(1.99)\end{array}$ & $\begin{array}{r}5.13^{\mathrm{a}} \\
(2.16)\end{array}$ & 0.0405 \\
\hline \# racimos & $\begin{array}{r}8.30^{\mathrm{a}} \\
(1.98)\end{array}$ & $\begin{array}{r}8.23^{\mathrm{a}} \\
(1.65)\end{array}$ & $\begin{array}{r}7.80^{\mathrm{a}} \\
(0.95)\end{array}$ & 0.5443 \\
\hline Longitud de la hoja & $\begin{array}{r}17.97^{\mathrm{a}} \\
(1.73)\end{array}$ & $\begin{array}{r}16.59^{\mathrm{a}} \\
(1.66)\end{array}$ & $\begin{array}{r}17.06^{\mathrm{a}} \\
(1.25)\end{array}$ & 0.1156 \\
\hline Diámetro de la hoja & $\begin{array}{r}22.21^{\mathrm{a}} \\
(2.35)\end{array}$ & $\begin{array}{r}22.02^{\mathrm{a}} \\
(2.07)\end{array}$ & $\begin{array}{r}21.95^{\mathrm{a}} \\
(1.80)\end{array}$ & 0.9449 \\
\hline \# de hojas & $\begin{array}{c}13.10^{\mathrm{b}} \\
(2.42)\end{array}$ & $\begin{array}{c}15.10^{\mathrm{ab}} \\
(3.35)\end{array}$ & $\begin{array}{c}17.27^{\mathrm{a}} \\
(3.01)\end{array}$ & 0.0019 \\
\hline \# de frutos por racimo & $\begin{array}{c}3.40^{\mathrm{a}} \\
(1.00)\end{array}$ & $\begin{array}{l}3.60^{\mathrm{a}} \\
(0.84)\end{array}$ & $\begin{array}{r}3.90^{\mathrm{a}} \\
(0.78)\end{array}$ & 0.1468 \\
\hline Peso del fruto & $\begin{array}{c}6.25^{\mathrm{a}} \\
(1.02)\end{array}$ & $\begin{array}{c}5.79^{\mathrm{a}} \\
(0.61)\end{array}$ & $\begin{array}{r}6.53^{\mathrm{a}} \\
(0.98)\end{array}$ & 0.1403 \\
\hline Rendimiento ton.ha ${ }^{-1}$ & $\begin{array}{r}10.42^{\mathrm{a}} \\
(1.70)\end{array}$ & $\begin{array}{r}9.66^{\mathrm{a}} \\
(1.02)\end{array}$ & $\begin{array}{r}10.88^{\mathrm{a}} \\
(1.63)\end{array}$ & 0.1158 \\
\hline Longitud del fruto & $\begin{array}{r}25.70^{\mathrm{a}} \\
(2.28)\end{array}$ & $\begin{array}{r}25.43^{\mathrm{a}} \\
(1.27)\end{array}$ & $\begin{array}{r}26.89^{\mathrm{a}} \\
(1.61)\end{array}$ & 0.0996 \\
\hline Diámetro del fruto & $\begin{array}{r}21.01^{\mathrm{a}} \\
(1.21)\end{array}$ & $\begin{array}{c}20.60^{\mathrm{a}} \\
(1.18)\end{array}$ & $\begin{array}{r}21.25^{\mathrm{a}} \\
(1.06)\end{array}$ & 0.3564 \\
\hline Acidez titulable & $\begin{array}{c}1.81^{\mathrm{b}} \\
(0.17)\end{array}$ & $\begin{array}{l}2.0^{\mathrm{a}} \\
(0.18)\end{array}$ & $\begin{array}{c}1.82^{\mathrm{b}} \\
(0.21)\end{array}$ & 0.0214 \\
\hline Solidos solubles & $\begin{array}{l}10.003^{\mathrm{a}} \\
(1.05)^{2}\end{array}$ & $\begin{array}{l}9.803^{\mathrm{a}} \\
(0.77)^{2}\end{array}$ & $\begin{array}{r}8.22^{\mathrm{b}} \\
(0.75)\end{array}$ & 0.00 \\
\hline Días total del ciclo & $\begin{array}{c}78.000^{\mathrm{a}} \\
(0.00)\end{array}$ & $\begin{array}{r}76.800^{\mathrm{a}} \\
(3.830)\end{array}$ & $\begin{array}{c}74.267^{\mathrm{b}} \\
(0.45)\end{array}$ & 0.0004 \\
\hline
\end{tabular}

$\overline{\text { Medias seguidas por la misma letra son estadísticamente iguales (Tukey 0.05). Los }}$ valores ( ) se refieren a la desviación estándar 
El número de frutos por racimo, peso de fruto, rendimiento por hectárea, longitud del fruto, diámetro del fruto, son estadísticamente iguales en las tres zonas de estudio (Tabla 2) pero al compararlos con los resultados de la caracterización morfológica de mora en Colombia realizada por Zamorano et al. (2007), se observa que los valores reportados son inferiores a los obtenidos en esta investigación, aunque el peso de fruto registrado en este trabajo es inferior al citado por Grijalba, Calderón y Pérez (2010) para la categoría de calidad extra estudiada en Colombia, aunque el diámetro y longitud de frutos de los tres pisos altitudinales estudiados en esta investigación, lo ubican en el calibre mediano según INEN (2010).

En cuanto a la forma, en los tres pisos altitudinales se encontraron solamente frutos de forma elíptica. El contenido de solidos solubles en los frutos del piso altitudinal bajo es inferior estadísticamente que en las otras dos zonas estudiadas con un valor promedio (8.22) similar al obtenido por Vásquez et al. (2006) que encontraron valores de 7.7 y $8.5^{\circ}$ Brix, pero en los tres pisos altitudinales se registra mayor contenido de solidos solubles que el reportado por Grijalba, Calderón y Pérez (2010) y Ayala, Valenzuela y Bohorquez (2013a). En el piso altitudinal medio, la acidez titulable fue estadísticamente mayor, sin embargo Ayala, Valenzuela \& Bohórquez (2013a) registraron valores superiores. Los frutos provenientes de las tres zonas estudiadas cumplen con los requisitos INEN (2010) en lo relativo al contenido de solidos solubles y acidez titulable, evidenciando la competitividad del producto local para el mercado en fresco y elaboración de procesados industriales. El contenido de solidos solubles podría considerarse como un parámetro ideal para el desarrollo de la industria local y nacional.
En el sector con el piso altitudinal más bajo utilizado se necesitó de menos días para pasar del estadio de botón cerrado a fruto maduro listo para el consumo, mientras que en el piso altitudinal alto, los días que transcurren de la floración a la cosecha son mayores. El número de días en los tres pisos fue cercano al registrado por Grijalba, Calderón y Pérez. (2010).

En cada uno de los pisos altitudinales se obtuvo prevalencia de una de las enfermedades que afectan significativamente la productividad del cultivo. En el piso medio se registra un porcentaje estadísticamente mayor de Botrytis que en el piso alto, pero en este piso el porcentaje de Oídio es estadísticamente superior que en los otros dos pisos altitudinales, entre los cuales no se encontró diferencia significativa; mientras que para Peronospora se registra el mayor valor en el piso bajo y estadísticamente diferente en los tres pisos altitudinales (Tabla 3). La prevalencia de Botrytis en la zona alta, Oídio en la media y Peronospora en la zona baja es estadísticamente significativa, ya que el P-value es inferior al nivel de significancia de 0.05 , lo que demuestra que la altura si afecta la incidencia de este tipo de enfermedades. En relación a la Peronospora que afectó la zona baja se observa que tiene el mayor valor de P-value (0.0243), demostrando que a un nivel de significancia de 0.01 se aceptaría la hipótesis nula, lo que indica que para la Peronospora no hay diferencia entre las medias de las tres poblaciones y la Peronospora afecta por igual a los tres pisos altitudinales. Esto también indica que este frutal puede producirse sin mayor contaminación por la aplicación de pesticidas, lo cual es un parámetro de calidad en la actualidad para el consumo directo y aprovechamiento industrial.

Tabla 3. Valores promedio de variables fitopatológicas evaluadas en la mora (R. glaucus) en los tres pisos altitudinales estudiados.

\begin{tabular}{lllll}
\hline \multirow{2}{*}{ Variable } & \multicolumn{3}{c}{ Piso altitudinal } & \multirow{2}{*}{ P-value } \\
\cline { 2 - 4 } & Alta & Media & Baja & 0.008 \\
Botrytis \% & $8.733^{\mathrm{b}}$ & $12.733^{\mathrm{a}}$ & $10.667^{\mathrm{ab}}$ & \\
& $(1.93)$ & $(3.16)$ & $(4.23)$ & 0.0003 \\
Oídio $\%$ & $8.1000^{\mathrm{a}}$ & $4.4667^{\mathrm{b}}$ & $4.7667^{\mathrm{b}}$ & \\
Peronospora \% & $(1.78)$ & $(3.46)$ & $(2.14)$ & 0.0243 \\
\hline
\end{tabular}

Medias seguidas por la misma letra son estadísticamente iguales (Tukey 0.05). Los valores ( ) se refieren a la desviación estándar. 


\section{Conclusiones}

No existe diferencia significativa entre la producción de ${ }^{\circ}$ de frutos y peso de frutos en los diferentes piso ecológicos. Sin embargo, los resultados sugieren que la mora Rubus glaucus Benth cultivada en el piso altitudinal bajo, es más resistente a enfermedades. Se sugiere establecer un sistema de seleccionan y propagación de solo las plantas con características superiores, que son identificadas muy fácilmente por los mismos productores pero que no ponen en práctica actualmente.

\section{Literatura citada}

Alzate, A.; Mayor, M.;Montoya, S.

2010. Influencia del manejo agronómico, condiciones edáficas y climáticas sobre las propiedades físicoquímicas y fisiológicas de la mora (Rubus glaucus BENTH.) En dos zonas de la región centro sur del Departamento de Caldas. Agron. 18 (2): 37 - 46.

Ayala , L.; Valenzuela , C.; Bohórquez, Y.

2013a. Caracterización Fisicoquímica de Mora de Castilla (Rubus glaucus Benth) en Seis Estados de Madurez. Biotecnología en el Sector Agropecuario y Agroindustrial, 11 (2): 10-18.

Ayala , L., Valenzuela, C., \& Bohórquez, Y.

2013b. Variables Determinantes de la Madurez Comercial en la Mora de Castilla (Rubus glaucus BENTH). Scientia Agroalimentaria, 1: 39-44.

Barrero Meneses, Luz Stella.

2009. Caracterización, evaluación y producción de material limpio de mora con alto valor agregado. Cundinamarca Colombia. Corpoica. 84 p.

Bernal J. y Díaz C.

2006. Materiales locales y mejorados de toamte de Árbol, Mora y Lulo sembrados por los agricultores y cultivares disponibles para su evaluacion en Colombia. Corpoica. C. I. La Selva. 14p.

Cancino, G., Sánchez, L, Quevedo, E.; Díaz, C.

2011. Caracterización fenotípica de accesiones de especies de Rubus L. de los municipios de Pamplona y Chitagá, región Nororiental de Colombia. Universitas Scientiarum, 16 (3): 219-233.

Franco, G., Giraldo, M. J.

2000. El cultivo de la mora. Corporación Colombiana de Investigación agropecuaria CORPOICA. Tercera edición. Manizales. 75 p.

Franco, T., \& Hidalgo, R.

2003. Análisis Estadístico de Datos de Caracterización Morfológica de Recursos Fitogenéticos. Boletín técnico no. 8, Instituto Internacional de Recursos Fitogenéticos (IPGRI), Cali, Colombia. 89p.

Garrido, P., Vásquez, W., \& Morillo, E.

2010. Análisis de la diversidad genetica de la mora cultivada (Rubus glaucus benth) y especies emparentadas en zonas productivas del Ecuador mediante marcadores moleculares. Quito, Ecuador. 12 p.

Grijalba, C., Calderón, L., \& Pérez, M.

2010. Rendimiento y Calidad de la Fruta en Mora de Castilla (Rubus glaucus Benth), Con y Sin Espinas, en Campo Abierto en Caji (Cundinamarca, Colombia). Revista de la Facultad Ciencias Básicas, 6 (1): 24-41.
Holdridge, LR. 1967. Life Zone Ecology. Tropical Science Center. San José, Costa Rica. 149 p.

INEN.

2010. Instituto Ecuatoriano de Normalización ,Norma Técnica Ecuatoriana, Frutas Frescas. Mora. Requisitos. Quito, Ecuador. 8 p.

Lemus, L.; Ramírez, N.

2002. Fenologia reproductiva en tres tipos de vegetación de la planicie costera de la peninsula de Paraguana, Venezuela. Acta Científica Venezolana, 53: 266-278.

MAGAP.

2013. La mora de Castilla. Quito.Ecuador. 20 p.

Martínez, A., Beltrán, O., Velastegui, G., Ayala , G., Yánez, W., \& Valle, L.

2007. Manual del Cultivo de la Mora De Castilla ( Rubus glaucus B). V y P Publicidad. Ambato, Ecuador. 51p.

Moreno, M., Villarrea, D., Lagos, T., Ordoñez, H., \& Criollo, H. 2011. Caracterización "In Situ” de Genotipos Silvestres y Cultivados de Mora Rubus spp en el Municipio de Pasto. Municipio De Pasto: Revista de Ciencias Agrícolas. 28 (2): 109 - 128.

Phillips, M., Arciniegas, A., Mata, A., \& Motamayor, J. 2012. Catálogo Clones de Cacao Selecionados por la CATIE para Siembras Comerciales. Turrialba, Costa Rica. 68 p.

Serna, P., Tamara R., L. M., Vallejo, I. y Galarza A. 2009. Perfil de mercado. Perfil de mora. CICO Centro de Inteligencia e Información Comercial. Quito, Ecuador. 23p.

Soria, N., Padilla, F., \& Larrea, G.

2014. Guía para el cultivo de mora de castilla (Rubus glaucus Benth). Quito: Editorial Politécnica-ESPE. Quito, Ecuador. 32p.

Valdés, J., Rodríguez, N., Velásquez, J., Sourd, D., González, G., Rodríguez, J., \& Rohde, W.

2012. Herramientas Para un Programa de Mejoramiento Genético del Guayabo (Psidium guajava L.) En Cuba. Agronomía Costarricense, 36 (2): 111-129.

Vásquez R., Ballesteros H., Muñoz C. y Cuellar M.

2006. Utilización de la abeja Apis mellifera como agente polinizador en cultivos comerciales de fresa (Fragaria chilloensis) y mora (Rubus glaucus) y su efecto en la producción. Editorial Produmedios. Bogotá-Mosquera (Cundinamarca). 77p.

Yih Huang, J., \& Ming Hu, J.

2009. Revision of Rubus (Rosaceae) in Taiwan. Taiwania, 54 (4): 285-310.

Zamorano, A., Cruz, A., Morillo, Y., Vásquez, H., \& Muños, J. 2007. Caracterización morfológica de mora en los departamentos de Valle del Cauca, Cauca y Nariño, de Colombia. Acta Agronómica, 56 (2): 51-60. 